\title{
A qualitative investigation of adherence to nutritional therapy in malnourished adult AIDS patients in Kenya
}

\author{
Filippo Dibari ${ }^{1,2, *}$, Paluku Bahwere ${ }^{1}$, Isabelle Le Gall ${ }^{3}$, Saul Guerrero' \\ David Mwaniki ${ }^{4,5}$ and Andrew Seal ${ }^{2}$ \\ 'Valid International, 35 Leopold Street, Oxford OX4 1TW, UK: ${ }^{2}$ UCL Centre for International Health and \\ Development, Institute of Child Health, 30 Guilford Street, London WCIN 1EH, UK: ${ }^{3}$ MSF-France, Nairobi, \\ Kenya/Paris, France: ${ }^{4}$ Centre for Public Health, Kenya Medical Research Institute, KEMRI/CPHR, Nairobi, \\ Kenya: ${ }^{5}$ Academy for Educational Development/Regional Office for Eastern and Central Africa, Nairobi, Kenya
}

Submitted 2 June 2010: Accepted 25 October 2010: First published online 4 February 2011

\begin{abstract}
Objective: To understand factors affecting the compliance of malnourished, HIV-positive adults with a nutritional protocol using ready-to-use therapeutic food (RUTF; Plumpy'nut ${ }^{\circledR}$ ).

Design: Qualitative study using key informant interviews, focus group discussions and direct observations.

Setting: Ministry of Health HIV/programme supported by Médecins Sans Frontièrs (MSF) in Nyanza Province, Kenya.

Subjects: Adult patients ( $n$ 46) currently or previously affected by HIV-associated wasting and receiving anti-retroviral therapy, their caregivers ( $n 2)$ and $\mathrm{MoH} / \mathrm{MSF}$ medical employees ( $n$ 8).

Results: Thirty-four out of forty-six patients were receiving RUTF $(8360 \mathrm{~kJ} / \mathrm{d})$ at the time of the study and nineteen of them were wasted $\left(\mathrm{BMI}<17 \mathrm{~kg} / \mathrm{m}^{2}\right)$. Six of the thirteen wasted out-patients came to the clinic without a caregiver and were unable to carry their monthly provision $(12 \mathrm{~kg}$ ) of RUTF home because of physical frailty. Despite the patients' enthusiasm about their weight gain and rapid resumption of labour activities, the taste of the product, diet monotony and clinical conditions associated with HIV made it impossible for half of them to consume the daily prescription. Sharing the RUTF with other household members and mixing with other foods were common. Staff training did not include therapeutic dietetic counselling.

Conclusions: The level of reported compliance with the prescribed dose of RUTF was low. An improved approach to treating malnourished HIV-positive adults in limited resource contexts is needed and must consider strategies to support patients without a caregiver, development of therapeutic foods more suited to adult taste, specific dietetic training for health staff and the provision of liquid therapeutic foods for severely ill patients.
\end{abstract}

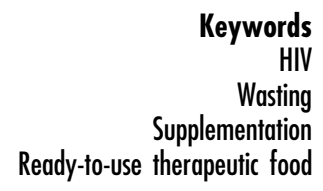

Undernutrition associated with HIV is a public health concern in Africa. Demographic and health surveys in eleven sub-Saharan countries estimated that $10 \cdot 3 \%$ of HIV-infected women (aged 15-49 years) had a BMI $<18.5 \mathrm{~kg} / \mathrm{m}^{2(1)}$; data for men were not available. In urban Lusaka, Zambia, $9 \%$ of adults (3624 out of 40778 ) started anti-retroviral therapy (ART) with a BMI $<16 \cdot 0 \mathrm{~kg} / \mathrm{m}^{2(2)}$.

Despite the increasing availability of ART, and patients enrolled in food programmes while on treatment reporting greater adherence to their medication ${ }^{(3,4)}$, HIV wasting is still common. Although the beneficial effects of ART on severe malnutrition in adults are well documented, Carr ${ }^{(5)}$ reported severe toxicity associated with ART in well-nourished individuals, and it has been suggested that side effects may be worse in malnourished individuals $^{(6)}$.

However, the relationship between nutritional status, therapy and survival of adults undergoing ART is controversial. According to Paton et al. ${ }^{(7)}$, a BMI $<17 \mathrm{~kg} / \mathrm{m}^{2}$ at the time of starting ART is associated with decreased survival in adults. A large observational study of patients receiving ART in Kenya and Cambodia ( $n$ 5069) showed that a weight gain of only $5 \%$ in 3 months increased survival in adults with a $\mathrm{BMI}<17 \mathrm{~kg} / \mathrm{m}^{2(8)}$.

Bahwere et $a l .{ }^{(9)}$ reported that a novel ready-to-use therapeutic food (RUTF), nutritionally similar to commercially 
available paediatric RUTF, was acceptable to malnourished Malawian patients who were not receiving ART, and that it improved their physical activity performance, nutritional status and survival.

On the other hand, Ndekha et al. ${ }^{(10)}$ found no difference in short-term survival when providing an energydense RUTF, or a lower-density porridge-based food, despite an increased weight gain with RUTF ( $n$ 450).

A retrospective analysis ${ }^{(11)}$ of surveillance data on adults under ART ( $n$ 329) and with a BMI $<17 \mathrm{~kg} / \mathrm{m}^{2}$, who were either receiving or not receiving an RUTF (Afya; Compact AS, Bergen, Norway), also reported that it was difficult to conclude a clear benefit of supplementary food in the early months of ART in terms of survival.

In 2007, the UN officially approved the use of RUTF for the treatment of acute malnutrition in children ${ }^{(12)}$. This approach has also been adopted in nutrition and health programmes in developing countries targeting adults with HIV/AIDS. However, only a paediatric formulation is commercially available and there is limited evidence for its efficacy, and little data on acceptability and adherence to this formulation in HIV/tuberculosis (TB)-positive adults.

To understand why nutritional support may not be providing consistent benefits, it is necessary to investigate, among other things, the compliance of patients to nutritional treatment and understand the factors that affect this. In the present paper, we use 'compliance' to describe the extent to which a person's behaviour taking medication, following a diet and/or executing lifestyle changes - corresponds to agreed recommendations from a health-care provider ${ }^{(13-15)}$.

The research questions of the present study aim (i) to understand and describe compliance with a protocol based on a specific RUTF (Plumpy'nut ${ }^{\circledR}$; Nutriset, Malaunay, France) among malnourished adults living with HIV; and (ii) to determine any key barriers to compliance.

To our knowledge, the present study is the first to qualitatively investigate compliance with nutritional protocols based on RUTF that aim at rehabilitating malnourished HIV adults. We report the results according to the COREQ (consolidated criteria for reporting qualitative research) guidelines ${ }^{(16)}$.

\section{Experimental methods}

The present research took place in Homa Bay, Nyanza Province, which is on the Kenyan side of Lake Victoria. In Kenya, in 2007, the prevalence of HIV was the highest in Nyanza Province $(15 \cdot 3 \%)$, more than double the national prevalence estimate ${ }^{(17)}$. In January 2006, the non-governmental organisation Médecins Sans Frontières (MSF), France, introduced a nutritional rehabilitation programme for malnourished adults enrolled in the Ministry of Health $(\mathrm{MoH})$ ART programme, Kenya, which utilised the most commonly available RUTF (brand name: Plumpy'nut ${ }^{\circledR} ; 8360 \mathrm{~kJ} / \mathrm{d}(2000 \mathrm{kcal} / \mathrm{d})$ equal to four sachets of $92 \mathrm{~g}$ each). Adults ( $\geq 15$ years of age) were considered malnourished (admission criteria) when BMI was $<17 \mathrm{~kg} / \mathrm{m}^{2}$ and/or middle upper-arm circumference (MUAC) was $<185 \mathrm{~mm}$ and/or the presence of oedema was observed. Discharge criteria from the nutrition programme included $\mathrm{BMI} \geq 18 \mathrm{~kg} / \mathrm{m}^{2}$ and $\mathrm{MUAC} \geq 185 \mathrm{~mm}$, and absence of oedema in at least two consecutive visits.

Along with the supply of RUTF, the medical staff provided generic nutritional counselling. These same staff members had never received any specific training in therapeutic nutritional treatment counselling for HIV adult patients.

\section{Subjects recruitment and sampling}

The study subjects, enrolled at the MoH/MSF HIV/TB programme in Homa Bay health district, Nyanza Province, Kenya, came from three groups: patients enrolled in the programme (some already nutritionally rehabilitated); their caregivers; and medical staff (counsellors, nurses and clinical officers). Patients $<15$ years of age were not admitted into the study group. The patients were recruited either at MoH/MSF HIV clinics A and B at Homa Bay hospital or from TB wards 7 and 8 of the same hospital.

The study followed a non-probabilistic, purposive, heterogeneous, non-proportional quota sampling system. Data saturation was achieved after approximately twothirds of the study; however, recruitment continued along the entire planned period (3 weeks). During the study period, patients coming for their usual routine visit were invited to participate by the nurse in charge of the nutrition programme. All the health staff working at the clinic during the study agreed to participate. All focus group discussions and interviews occurred in a quiet area within the HIV clinic compound in the presence of researchers only, whereas direct observations were made in the TB multidrug resistance ward at Homa Bay hospital.

\section{Data collection and data analysis}

The research applied three qualitative methods and the results were triangulated. Focus group discussions involved patients and caregivers and one separate session with members of the MoH/MSF medical staff; semi-structured interviews were administered to patients on a one-toone basis; direct unobtrusive observations were made of Plumpy'nut ${ }^{\circledR}$ distribution and consumption in the HIV/TB wards. The questionnaires and focus group discussions used a variety of techniques, including free listing, ranking exercises and open-ended questions (see footnotes in Table 3).

\section{Questionnaires and focus group guides}

Two guides were developed and piloted for use in the focus groups: one for patients and caregivers and one for the health staff members. The guides and questionnaires covered four topics: (i) information provided to patients about the recommended use and consumption of Plumpy'nut ${ }^{\circledR}$; (ii) knowledge and attitude of patients about the role of 
Plumpy'nut ${ }^{\circledR}$ in their therapy; (iii) dietary practices and Plumpy'nut ${ }^{\circledR}$ consumption; and (iv) patient's and caregiver's experience of the Plumpy'nut ${ }^{\circledR}$ distribution system.

A checklist for the direct observations was developed and covered the ways of consuming Plumpy'nut ${ }^{\circledR}$, their advantages and disadvantages, together with the role of the caregiver.

All focus group discussions were recorded on tape, allowing the checking of information that was not clear from the written notes. The focus group discussions and interviews did not last for more than 1.5 and $1 \mathrm{~h}$, respectively. The direct observation sessions lasted between $30 \mathrm{~min}$ and $1 \mathrm{~h}$. A small number of photographs were taken with the informed consent of patients.

\section{The research team and the relationship with subjects}

The focus group discussions and interviews were conducted in the Luo language. Two native speakers, a female and a male, were recruited as interpreters and provided with $2 \mathrm{~d}$ training on the study and methods to be used. Time for familiarisation with the study guides was provided.

The principal researcher was a Caucasian male who was trained in public health nutrition at MSc level, and was not an MSF employee. He attended all focus group sessions and benefited from simultaneous translation by an interpreter. The principal researcher personally facilitated, in English, the focus group discussion with the health staff, and subsequently transcribed the recorded tape himself. Other discussions were facilitated by the study interpreters. The discussion transcript was not returned to the members for comments or corrections. All participants were provided with information about the study and assured that clinical services or RUTF provision would not be affected by refusal to participate.

\section{Identification of common themes}

The focus group discussion transcripts were manually coded by the principal researcher, highlighting keywords, key concepts and any minor or contradictory themes. Records of direct observations were reviewed and evidence summarised. Quantitative data from the interviews and the clinical and socio-economic profile of the focus group participants were entered in EpiInfo version $3 \cdot 4 \cdot 3$ (Centers for Disease Control and Prevention, Atlanta, GA, USA) and analysed in the STATA statistical software package version $8 \cdot 0$ (Stata Corp., College Station, TX, USA).

Detailed tables were prepared for each identified theme and its associated sub-themes. The theme tables contained separate columns referring to the evidence obtained on each theme using one of the three research methods. This allowed triangulation of evidence and comparison and discussion of the common themes that emerged. Following this process, the themes and sub-themes from the separate tables were compiled and overall conclusions drawn. Conclusions and associated recommendations were discussed with the health and management staff of the programme during a feedback process.

\section{Results}

From January 2008 to March 2009, MSF admitted 782 malnourished adults into the therapeutic nutrition programme; the monthly mean weight gain was $1 \cdot 8$ (SD 0.5 ) and $1.8(\mathrm{sD} 0 \cdot 6) \mathrm{g} / \mathrm{kg}$ per $\mathrm{d}$, respectively, for severely and moderately malnourished adults.

\section{Study group characteristics}

Table 1 provides descriptive data on the forty-six participating patients. Thirty-four were still under treatment with Plumpy'nut ${ }^{\circledR}$. More than half of them were women and the average age was 33 years. All patients were married and the majority had their spouse still alive; twelve out of the forty-six patients were widowers. All subjects who were approached agreed to participate in the study and provided written informed consent.

Table 2 summarises the methods used and the number of patients, caregivers and health staff participants. Twenty-two current and ex-patients received one-to-one interviews and eighteen participated in focus group discussions. Six in-patients were directly observed. Two caregivers were recruited to participate in focus group discussions. Eight MoH/MSF employees participated in the focus groups with the medical staff (counsellors, nurses and medical doctors). None of the subjects were involved in more than one data collection method.

Table 1 Profile of participating patients ( $n$ 46)

\begin{tabular}{|c|c|c|}
\hline \multirow[b]{2}{*}{ Characteristic } & \multicolumn{2}{|c|}{ Participants } \\
\hline & $n$ & $\%$ \\
\hline Male & 18 & 39 \\
\hline Female & 28 & 61 \\
\hline Age (years) & \multicolumn{2}{|c|}{$33 \cdot 3$} \\
\hline Married (missing records $=1$ ) & 45 & 100 \\
\hline Spouse alive & 33 & 73 \\
\hline Widowers & 12 & 27 \\
\hline $\begin{array}{l}\text { Mean number of children (missing } \\
\text { records }=1)^{\star}\end{array}$ & \multicolumn{2}{|c|}{$3 \cdot 0$} \\
\hline \multicolumn{3}{|l|}{ Serological status (missing records $=6$ ) } \\
\hline HIV positive & 24 & 60 \\
\hline HIV/TB positive & 16 & 40 \\
\hline \multicolumn{3}{|l|}{$\begin{array}{l}\text { Nutritional rehabilitation (missing } \\
\text { records = 1) }\end{array}$} \\
\hline \multicolumn{3}{|l|}{$\begin{array}{l}\text { Enrolled into the nutrition programme at } \\
\text { the time of the study }\end{array}$} \\
\hline $\begin{array}{l}\text { No longer meeting admission criteria } \\
\text { of malnutritiont }\end{array}$ & 15 & 33 \\
\hline $\begin{array}{l}\text { Meeting admission criteria of } \\
\text { malnutrition } \ddagger\end{array}$ & 19 & 42 \\
\hline Discharged by the time of the study & 11 & 25 \\
\hline
\end{tabular}

TB, tuberculosis; MUAC, middle upper-arm circumference.

*Age not specified.

$+B M I \geq 17 \mathrm{~kg} / \mathrm{m}^{2}$ and $/$ or MUAC $\geq 185 \mathrm{~mm}$.

$\ddagger$ This group included thirteen out-patients and six in-patients. The in-patients were from the TB multi-drug resistance ward. 
Table 2 Summary of methods and participants

\begin{tabular}{lc}
\hline Methods & $\begin{array}{c}\text { Participants } \\
(n \text { 56 })^{*}\end{array}$ \\
\hline Individual interviews with current and ex-patients & 22 \\
Five focus group discussions from three to five & \\
participants, including: & 18 \\
$\quad$ Current and ex-patients & 2 \\
$\quad$ Caregivers & 6 \\
Direct observations on individual in-patients and their & 8 \\
caregiver's role & 8 \\
\hline
\end{tabular}

*None of the study subjects participated in more than one method.

Among the out-patients ( $n$ 13) who were still malnourished, six travelled to the clinic without a caregiver on the day of the study (two missing data). One walked, whereas the rest took public transport; the average time to reach the clinic was almost $2 \mathrm{~h}$. The six in-patients recruited for the direct observations were found in the TB wards of Homa Bay hospital and they were all HIV positive; two of them were multi-drug resistant and their appetite might have been affected by their TB status and/or HIV status, plus by side effects of specific drugs.

\section{Understanding compliance}

The following sections summarise the key themes emerging from the analysis of the results. Only fourteen out of twenty-two interviewed patients reported complying with the prescribed amount of Plumpy'nut ${ }^{\circledR}$.

\section{Perceptions about Plumpy'nut ${ }^{R}$}

Table 3 lists the aspects that may both enhance or decrease compliance with Plumpy'nut ${ }^{\circledR}$, and were mentioned during the majority of focus groups and interviews. It was frequently reported that Plumpy'nut ${ }^{\circledR}$ can be 'associated with a drug' (same role and effect of a drug), 'brings (physical) strength' and 'allows to go back to work'. Increases in weight gain were reported and feelings of hunger said to decrease. Positive feelings about the use of the product can be summarised by the quote: 'ART is a drug to fight the infections, but does not give strength like Plumpy'nut ${ }^{\circledR}$ to go back to work'.

Turning to the negative perceptions of participants, some patients complained that the taste of Plumpy'nut ${ }^{\circledR}$ was responsible for nausea and vomiting. Participants also argued that the 'first 3 or $4 \mathrm{~d}$ are the most critical ones' and that 'after then, it becomes easier' to comply with the prescription. Among the patients interviewed, only one out of forty mentioned oral thrush as the main cause for low acceptability. In contrast, the health staff focus group voiced that the initial clinical conditions including swallowing capacity are crucial for patients' compliance. The interviewees provided suggestions about how to improve the product (see Table 3 ).

The medical staff expressed doubts about the role of Plumpy'nut ${ }^{\circledR}$ in promoting weight gain, since 'ART is far more important in severely malnourished patients'.
Sharing practices

Compliance was found to be closely linked with foodsharing practices. More than half (fourteen out of twentytwo) of the interviewed patients reported sharing Plumpy'nut ${ }^{\circledR}$ with children and other adults and this was confirmed by most focus groups. The medical staff was particularly concerned about the sharing practice at the community level, because of their observation that '... in the hospital wards, it is common'. In two of the focus groups, patients reported incidents of Plumpy'nut ${ }^{\circledR}$ trading or selling. This, however, seems to be limited to schoolchildren, because among adults the product was associated with HIV treatment and was thus potentially stigmatising. The majority of patients who declared that they tried to prevent sharing ('hiding the product in the closet from children'; '...or from adults') did so because Plumpy'nut ${ }^{\circledR}$ was considered part of the 'medical drug prescription'. Only one interviewee reported that Plumpy'nut ${ }^{\circledR}$ could be actually harmful for an HIV-negative person. One member of the medical staff suggested that Plumpy'nut ${ }^{\circledR}$ is so important for household food security that sharing represents a strategy to delay the moment of programme discharge.

\section{Mixing Plumpy'nut ${ }^{(B)}$ with other foods}

Only one patient reported consuming Plumpy'nut ${ }^{\circledR}$ exclusively, whereas mixing Plumpy'nut ${ }^{\circledR}$ with other food was a common practice mainly with local staple starchy food (ugali), fresh vegetables, fish, rice, cereals, legumes, meat, cooked vegetables (sukuma wiki) and chapatti (in order of reported frequencies). Monotony of diet, nausea, vomiting or salty taste were the main reasons for mixing. Stirring Plumpy'nut ${ }^{\circledR}$ into hot water produces something that is similar to a popular, peanut-based, traditional food (ogila). Some others mentioned that 'once you start mixing the Plumpy'nut ${ }^{\circledR}$, it is hard to go back and eat it alone'. Members of the medical staff suggested (contrary to programme recommendations) that patients with severe clinical conditions (e.g. oral thrush) should mix the Plumpy'nut ${ }^{\circledR}$ with tea.

\section{Key barriers to compliance}

\section{Inability to transport ration}

Physical weakness, absence of a caregiver during collection of supplies, cost of transport and stigma were key barriers to compliance. The prescribed supply of Plumpy'nut ${ }^{\circledR}$ per out-patient was monthly and weighed approximately $12 \mathrm{~kg}$. A malnourished out-patient without a caregiver did not have enough strength to carry such weight. Therefore, the out-patients were invited to take half of the monthly ration and come back to collect the second half of the supply in 2 weeks' time. Health staff members reported that most patients could not afford to travel twice a month to the clinic. It can be speculated that these patients spread out the 2 weeks' provision along the entire month. This is consistent with the reports of many patients. No data could be collected on how many 
Table 3 Summary of key themes and sub-themes

\begin{tabular}{ll}
\hline Theme & $\begin{array}{l}\text { Individual } \\
\text { interviews }\end{array}$ \\
\hline
\end{tabular}

Compliance with Plumpy'nut ${ }^{\circledR}$

- Only approximately half of the patients complied with the prescribed amount

Positive aspects reported about Plumpy'nut ${ }^{\circledR}$ - participants think that:

- It is similar to a drug rather than a food, in terms of both usage and role in recovery

- It 'brings strength'

- It 'allows to go back to work'

- Increases weight gain

- Decreases the feeling of hunger

- Has a smell and packaging that are well accepted

- Offers the possibility to mix it with other food and therefore reduce:

$\bigcirc$ diet boredom

$\circ$ nausea

Negative aspects reported about Plumpy'nut ${ }^{\circledR}$ - participants think that:

- It can cause nausea and vomiting

- The first 3-4 d of consumption are crucial for compliance, becoming easier later

- The taste is:

$\circ$ too sweet

O too oily

$O$ too salty

- Consistency should be more liquid or like a biscuit or a powder (milk powder)

- It comprises a monotonous diet, leading to boredom

Sharing Plumpy'nut ${ }^{\circledR}$ with both other adults and/or children is a common practice

Reasons for sharing:

- Food insecurity in the household

- Children like it

- The partner or relative is ill and/or HIV positive but not malnourished (energy booster)

Mixing Plumpy'nut ${ }^{\circledR}$ with foods is a common practice

Reasons for mixing:

- To reduce monotony of the diet

- To reduce nausea, vomit, salty taste

- Because the Plumpy'nut ${ }^{\circledR}$ has separated into oil and solid phases

- Because Plumpy'nut ${ }^{\circledR}$ with water reminds participants of a traditional food

- Because it was suggested by the health staff

Knowledge and attitudes of medical staff

Medical staff expressed doubts about the positive role played by Plumpy'nut ${ }^{\circledR}$ in nutritional rehabilitation; ART was perceived as being much more important

Patient counselling

- Counselling messages focus on:

$\bigcirc$ human nutrition (e.g. 'proteins are available in meat, eggs and cheese')

$\bigcirc$ improvement of general conditions when consuming Plumpy'nut ${ }^{\circledR}$ (weight gain, appetite, strength)

- Most patients did not know the relationship that exists between HIV infection, their thinness and their ART therapy

- Staff declared that they did not know what counselling to provide to patients with severe clinical conditions (e.g. oral thrush)

- Most patients do not receive routine information about why, when and how to consume Plumpy'nut ${ }^{\mathbb{B}}$; when this happens, it comes from the individual initiative of the health staff

Distribution system for Plumpy'nut ${ }^{\circledR}$

- Half of the patients still under nutritional rehabilitation come to the HIV clinic without a caregiver

- The patients cannot carry more than a 2 weeks' supply of Plumpy'nut ${ }^{\circledR}(\sim 6 \mathrm{~kg})$ instead of the whole month's supply

- The appointment schedule for ART or clinical check-ups is monthly in most cases; therefore, patients or caregivers do not come back to collect the missing 2 weeks' supply of Plumpy'nut ${ }^{\circledR}$

- Very weak patients are in absolute need of the caregiver even to open the sachets, to mix it with other food (when needed) and consume it

- Bulky supply (6-12 kg) and branded container (box) are very noticeable and associated with stigma within the community

ART, anti-retroviral therapy.

*The focus groups involved either patients together with caregivers (five groups, with three to five participants) or health staff members (one group with eight participants).

†Tool used: closed and/or open questions.

$\ddagger$ Tool used: free listing.

$\S$ Tool used: ranking exercise to select the main themes reported here. 
patients came back to collect the missing mid-month supply or the relationship with defaulting and survival.

\section{Stigma associated with therapeutic foods}

In a region where HIV-related secondary malnutrition is common and treated with Plumpy'nut ${ }^{\circledR}$, this product has become strongly associated with HIV. For this reason many patients were reluctant to walk home with boxes reading the brand name (Plumpy'nut ${ }^{\circledR}$ ). One patient said 'I can disguise the ART tablets saying that they cure something else, but I cannot disguise Plumpy'nut ${ }^{\circledR}$ because a month's supply is too bulky and visible'.

\section{Clinical status and role of the caregiver}

Among the in-patients who were directly observed, all patients with a severe clinical condition could not comply with the prescribed amount. Two of them died during the course of the study. Alternative therapeutic diets, such as therapeutic liquid milk (formulas F75 and F100), the intake of which may have been easier for severely ill patients, was not available.

It was reported and observed that in-patients in the ward with the most severe clinical conditions can consume Plumpy'nut ${ }^{\circledR}$ only if mixed with other suitable food (e.g. water or porridge). This requires assistance from the caregiver. Patients with severe clinical conditions were so weak that they did not have sufficient strength even to open the sachet and mix the product with food.

\section{Discussion}

The present study has indicated that compliance with therapeutic feeding within this HIV programme is likely to be low. We have identified a number of issues that may reduce the consumption of RUTF to below the prescribed level. Although the nature of the study setting might have affected the patients' response, some of the findings are likely to be generalisable to other HIV and adult nutrition programmes that use this specific therapeutic food and a similar nutritional protocol.

The present study identified two groups of factors that limit specifically an RUTF-based protocol compliance: (i) factors directly related to the product design and (ii) factors related to programme design.

\section{Factors related to product design}

Although widely used for other patient groups, it is important to note that Plumpy'nut ${ }^{\circledR}$ was designed for treating severe acute malnutrition in children. There is evidence $^{(18)}$ to suggest that reported symptoms such as nausea and vomiting when consuming RUTF could be related to the drug treatment too. The taste of Plumpy'nut ${ }^{\circledR}$ was not considered suitable by many adult patients and therefore they often reduced intake or mixed it with other food. This practice may compromise the efficacy of RUTF by reducing overall energy and nutrient intake and delaying nutritional rehabilitation.
Despite the problems of taste and consistency acceptability, the results of this research suggested that most patients valued the nutritional therapy provided by Plumpy'nut ${ }^{\circledR}$. Their attitude towards the product was more positive than that of most medical employees, perhaps because of lack of staff training on the anticipated benefits. However, the interviewed patients would reduce the sweetness (somebody suggested the addition of lemon juice), and would like to change the consistency into something 'more liquid' or 'into the form of a biscuit' or powder. Rarer comments mentioned included suggestions for improving the packaging, such as the sachet packaging should be replaced with ice-cream cup with lid'.

\section{Factors related to programme design}

Certain problems in accessing and consuming the therapeutic food were accentuated by the design of the overall programme and inappropriate staff training. Patients were not informed about how to correctly consume the RUTF. For example, counselling messages inappropriately advised adding the RUTF to tea and advised feeding strategies that were not feasible for the patient. Advice to consume RUTF at meal times (3 times/d) and/or together with ART drugs was not conducive to ensuring adequate intake. Some of the patients admitted that they disregarded such advice because of the 'strong' - difficult to eat - nature of the product, their bad clinical conditions combined with ART side effects (lack of appetite, nausea, vomiting, etc.). More appropriate advice would have been that based on therapeutic feeding guidelines ${ }^{(19)}$ and should have recommended having small bites of RUTF throughout the day until the daily prescription of sachets was consumed. Box 1 presents the most common questions expressed by

\section{Box 1}

During dietetic counselling* sessions, the topics to be covered should respond to the following questions expressed by patients during the study: What is Plumpy'nut ${ }^{\circledR}$ (food/drug; composition)? Who is supposed to use it? What are its components? How often should I eat it along the day? What should I do when I do not manage to eat it because of nausea, vomiting or lack of appetite? Could I mix it with other foods? What should I avoid to drink or eat together with Plumpy'nut ${ }^{\circledR}$ ? What are the consequences if I share my ration of Plumpy'nut ${ }^{\circledR}$ with other persons? Is it good for somebody who is not HIV positive to eat Plumpy'nut ${ }^{\circledR}$ ? Is it good for somebody who is not thin to eat Plumpy'nut ${ }^{\circledR}$ ? When should I stop eating Plumpy'nut ${ }^{\circledR}$ ? Will I become thin again when I stop eating it?

*For an example of dietetic counselling material for HIV therapeutic feeding in adults, contact filippo@validinternational.org. 
patients during the present study. The same questions lead to key counselling messages.

Frail patients who came to a clinic appointment without a caregiver sometimes ended up losing half of the prescribed RUTF supply, as they were unable to transport the food to their home. The situation could be improved by re-design of the distribution system, perhaps involving community-based organisations to support home-based care. Their importance is recognised in many HIV and nutrition interventions and protocols ${ }^{(20-24)}$. The health programme also needed to acknowledge and address the stigma factor attached to Plumpy'nut ${ }^{\circledR}$. This could involve the use of anonymous packaging.

In case of extremely frail patients, the caregiver played a fundamental role in trying to overcome key barriers for compliance, such as simply opening the sachets or mixing it with other foods.

\section{Main limitations of the present study}

The present research did not correlate the collected data with clinical information such as patients' survival, CD4 count, weight gain or BMI changes that might have helped in understanding the impact of such a programme in the presence of household/community sharing, food mixing, lack of counselling and clinical difficulties in swallowing among others.

The present research did not aim to understand the link between nutritional therapy and ART compliance. RUTF is meant to be used in out-patient programmes, but the present study was restrained by lack of observations at the community level.

The two study interpreters were not only employed as health promoters in the $\mathrm{MoH}$ HIV programme but were also clients of the MoH/MSF HIV/TB programme. One of them had been previously successfully rehabilitated in the nutritional programme. Therefore, they might have previously met some of the patients enrolled in the study and/or developed specific perceptions about the topic, introducing possible bias.

\section{Conclusions}

Overall, the research suggests that training and information material is needed to ensure that medical staff can support the patient to overcome the first most crucial phase of the treatment. The very first contact with the patient should include a test to assess the swallowing capacity. Reduced capacity to swallow is common and it may imply a lower compliance with nutritional therapy and an increase in mortality risk. Consistent with the community-based management of malnutrition approach, medical staff should ask the patient to consume some amount of RUTF in his presence to verify his swallowing capacity. According to the results of this test, dietetic solutions must be discussed and agreed upon together with the patient and the caregiver when present. Patients who are unable to swallow at all should be hospitalised and treated according to the in-patient nutritional therapeutic protocol developed by $\mathrm{WHO}^{(25)}$, which utilises milk-based liquid therapeutic nutritional products such as F75 and F100.

The study showed that Plumpy'nut ${ }^{\circledR}$, designed for malnourished children, needs improvement to meet the palatability preferences expressed by adult patients. A reduction in the perception of sweetness and fat components was solicited by the patients. However, any $\mathrm{HIV} / \mathrm{TB}$ therapeutic nutrition programme should not introduce newly designed RUTF until positive clinical results have been demonstrated.

Further studies more closely correlating compliance and recovery rates are likely to improve the perception of medical staff towards the effectiveness of this kind of programme, possibly improving their performance as well.

\section{Acknowledgements}

The present study was funded by Valid Nutrition via a grant from Irish International Cooperation. Valid Nutrition is involved in the development of ready-to-use therapeutic foods for use in AIDS patients. The authors have no conflict of interest to declare. F.D. conceptualised the study, collected and analysed the data and wrote the draft manuscript; P.B. and S.G. assisted in study design and interpretation of the data; I.L.G. and D.M. facilitated data collection and supported the fieldwork; A.S. provided overall research supervision and contributed to the study design and to later versions of the manuscript. Other contributions came from Lio Fieschi and Nicky Dent who gave useful technical advice. The authors express deep appreciation to the study team, the participants and the staff of the MSF-France and Homa Bay TB multi-drugs resistance ward for their support, and in particular to Pamela Pomito.

\section{References}

1. Uthman OA (2008) Prevalence and pattern of HIV-related malnutrition among women in sub-Saharan Africa: a metaanalysis of demographic health surveys. BMC Public Health 8, 226-230.

2. Koethe JR, Lukusa A, Giganti MJ et al. (2010) Association between weight gain and clinical outcomes among malnourished adults initiating antiretroviral therapy in Lusaka, Zambia. J Acquir Immune Defic Syndr 53, 507-513.

3. Wanke CA, Silva M, Knox TA et al. (2000) Weight loss and wasting remain common complications in individuals infected with human immunodeficiency virus in the era of highly active antiretroviral therapy. Clin Infect Dis 31, 803-805.

4. Byron E, Gillespie S \& Nangami M (2006) Integrating nutrition security with treatment of people living with HIV: lessons from Kenya. Food Nutr Bull 29, 87-97.

5. Carr A (2003) Toxicity of antiretroviral therapy and implications for drug development. Nat Rev Drug Discov 2, 624-634. 
6. Koethe JR, Chi BH, Megazzini KM et al. (2009) Macronutrient supplementation for malnourished HIV-infected adults: a review of the evidence in resource-adequate and resource-constrained settings. Clin Infect Dis 49, 787-798.

7. Paton NI, Sangeetha S, Earnest A et al. (2006) The impact of malnutrition on survival and the CD4 count response in HIV-infected patients starting antiretroviral therapy. $H I V$ Med 7, 323-330.

8. Madec Y, Szumilin E, Genevier C et al. (2009) Weight gain at 3 months of antiretroviral therapy is strongly associated with survival: evidence from two developing countries. AIDS 27, 853-861.

9. Bahwere P, Sadler K \& Collins S (2009) Acceptability and effectiveness of chickpea sesame-based ready-to-use therapeutic food in malnourished HIV-positive adults. Patient Prefer Adberence 3, 67-75.

10. Ndekha MJ, van Oosterhout JJ, Zijlstra EE et al. (2009) Supplementary feeding with either ready-to-use fortified spread or corn-soy blend in wasted adults starting antiretroviral therapy in Malawi: randomised, investigator blinded, controlled trial. BMJ 338, b1867-b1875.

11. Sauvageot D, Huerga H, Genevier C et al. (2007) Does ready-to-use food supplementation (Afya) improve shortterm outcomes in HIV-infected malnourished adults commencing ART in rural Kenya? Lessons learned. http:// www.epicentre.msf.org/about-us/folder.events/2007-epicentrescientific-day/

12. World Health Organization, World Food Programme, United Nations Standing Committee on Nutrition et al. (2007) Community-Based Management of Severe Acute Malnutrition. Geneva: WHO.

13. Rand CS (1993) Measuring adherence with therapy for chronic diseases: implications for the treatment of heterozygous familial hypercholesterolemia. Am J Cardiol 72, 68D-74D.
14. Haynes RB (1979) Determinants of Compliance: The Disease and the Mechanics of Treatment. Baltimore, MD: John Hopkins University Press.

15. World Health Organization (2003) Adherence to Long-term Therapies: Evidence for Action. Geneva: WHO.

16. Tong A, Sainsbury P \& Craig J (2007) Consolidated criteria for reporting qualitative research (COREQ): a 32-item checklist for interviews and focus groups. Int J Qual Health Care 19, 349-357.

17. National AIDS and STD Control Programme (2007) Kenya AIDS Indicator Survey 2007: Preliminary Report. Nairobi: NASCOP (Ministry of Health, Kenya).

18. Food and Nutrition Technical Assistance Project (2004) HIV/AIDS: A Guide for Nutritional Care and Support, 2nd ed. Washington, DC: Academy for Educational Development; available at http://www.fantaproject.org/downloads/ pdfs/HIVAIDS_Guide02.pdf

19. Valid International Concern Worldwide (2006) Communitybased Therapeutic Care (CTC): A Field Manual. Oxford: Valid International.

20. Family Health International (2007) HIV, Nutrition and Food: A Practical Guide for Technical Staff and Clinicians. Durham, NC: FHI.

21. Piwoz E (2004) Nutrition and HIV/AIDS: Evidence, Gaps and Priority Actions. Washington, DC: SARA, FANTA.

22. Food and Nutrition Technical Assistance Project (2008) Ethiopia Nutrition and HIV Tools. Washington, DC: FANTA.

23. Food and Nutrition Technical Assistance Project (2008) Nutrition Care for People Living with HIV and AIDS: Training Manual for Community and Home-Based Care Providers Facilitators Guide and Participant Handouts. Washington, DC: FANTA.

24. Bonnard P (2002) HIV/AIDS Mitigation: Using What We Already Know. Technical Note. Washington, DC: FANTA.

25. World Health Organization (1999) Management of Severe Malnutrition: A Manual for Physicians and Other Senior Health Workers. Geneva: WHO. 